\title{
LACUNARY DIRECTIONAL MAXIMAL FUNCTION ON THE HEISENBERG GROUP
}

\author{
JOONIL KIM
}

\begin{abstract}
We prove the $L^{p}$ boundedness of the maximal function along a family of lines $\left\{t\left(2^{k_{1}}, 2^{k_{2}}, 2^{k_{3}}\right)\right\}$ on the Heisenberg group $\mathbb{H}^{1}$. The proof is based on the group Fourier transform and the angular Littlewood-Paley decompositions in the Heisenberg group in [7].
\end{abstract}

\section{Introduction}

Let $K=\left(k_{1}, \cdots, k_{d}\right) \in \mathbb{Z}^{d}$, and let $v_{K}=\left(2^{k_{1}}, \cdots, 2^{k_{d}}\right)$. Then we define a maximal function for $f \in L_{l o c}^{1}\left(\mathbb{R}^{d}\right)$ :

$$
M_{d}^{l a c} f(x)=\sup _{K \in \mathbb{Z}^{d}, r>0} \frac{1}{2 r} \int_{-r}^{r} f\left(x-\left(t v_{K}\right)\right) d t .
$$

The operator $M_{d}^{\text {lac }}$ was studied involving the differentiation in lacunary directions. The $L^{p}$-boundedness of $M_{2}^{\text {lac }}$ with $p \geq 2$ was proved by use of the covering lemma in [4] and [11]. For the proof of the full range $p>1$, the Fourier transform and the Littewood-Paley theory on angular sectors were successfully used in [9]. Furthermore this result can be extended to any $d \in \mathbb{Z}^{+}$ in [1]. Recently in [6], the $L^{p}$ theory is made for the curve $\left(2^{k_{1}} p(t), 2^{k_{2}} p(t)\right)$ with $p$ a polynomial. In this paper we study the analogue of $M_{3}^{\text {lac }}$ on the Heisenberg group $\mathbb{H}^{1}$ as a variable coefficient model. Let $\mathbb{H}^{1}$ be the Heisenberg group identified with $\mathbb{R}^{2} \times \mathbb{R}^{1}$ endowed with the group multiplication: $(p, q, t) \cdot\left(p^{\prime}, q^{\prime}, t^{\prime}\right)=\left(p+p^{\prime}, q+q^{\prime}, t+t^{\prime}+2\left(p \cdot q^{\prime}-p^{\prime} \cdot q\right)\right)$. Let us define a maximal function for $f \in L_{l o c}^{1}\left(\mathbb{H}^{1}\right)$ :

$$
\mathcal{M}^{l a c} f(x)=\sup _{K \in \mathbb{Z}^{3}, r>0} \frac{1}{2 r} \int_{-r}^{r} f\left(x \cdot\left(t v_{K}\right)^{-1}\right) d t .
$$

The purpose of this paper is to prove Theorem 1.

Theorem 1. For $1<p \leq \infty,\left\|\mathcal{M}^{l a c} f\right\|_{L^{p}\left(\mathbb{H}^{1}\right)} \leq C_{p}\|f\|_{L^{p}\left(\mathbb{H}^{1}\right)}$ with a constant $C_{p}$.

Received October 19, 2003.

2000 Mathematics Subject Classification. Primary 42B20,42B25. 
Let $K=\left(0, k_{2}, k_{3}\right)$ with $k_{2}, k_{3} \in \mathbb{Z}$ and $\varphi_{j}(u)=\varphi\left(u / 2^{j}\right) / 2^{j}$ with $\varphi \in$ $C^{\infty}[1,2]$. We define a measure $\mu_{j, K}$ on $\mathbb{R}^{3}$ by

$$
\mu_{j, K}(f)=\int f\left(\left(1,2^{k_{2}}, 2^{k_{3}}\right) s\right) \varphi_{j}(s) d s .
$$

Let us use the change of variable $t=2^{k_{1}} s$ in (1.2). Then Theorem 1 is proved by the following $L^{p}$ estimate for the three parameter maximal function $\sup _{j, K} \mu_{j, K} *$ $f$.

$$
\left\|\sup _{j, K} \mu_{j, K} * f\right\|_{L^{p}\left(\mathbb{H}^{1}\right)} \lesssim\|f\|_{L^{p}\left(\mathbb{H}^{1}\right)} .
$$

In [7] the $L^{p}$ estimate of the maximal function on the flat curve

$$
\Gamma(t)=(t, \gamma(t), t \gamma(t))
$$

in $\mathbb{H}^{1}$ is made under the convexity condition of $\gamma$ and the doubling condition such that $\gamma^{\prime}(C t) \geq 2 \gamma^{\prime}(t)$. This doubling condition of $\gamma^{\prime}$ has the essentially same feature as the lacunary direction of $v_{K}$. The normal directions of $v_{K}$ 's are handled by the angular Littlewood-Paley decompositions in [7]. The complements of these normal directions are controlled by the majorization of the strong maximal function and the decay property of frequency variables. To adapt this scheme (already known on the Euclidean space in [1] and [9]) on $\mathbb{H}^{1}$, we need to discuss the Fourier transform of the measure $\mu_{j, K}$ on the Heisenberg group $\mathbb{H}^{1}$. The group Fourier transform of $\widehat{f}$ with $f \in L^{1}\left(\mathbb{H}^{1}\right) \cap L^{2}\left(\mathbb{H}^{1}\right)$ is defined as an operator-valued function from $\mathbb{R}^{1}$ to the space of bounded operators on $L^{2}\left(\mathbb{R}^{1}\right)$. For $\lambda \in \mathbb{R}^{1}, \widehat{f}(\lambda)$ is the operator defined on $L^{2}\left(\mathbb{R}^{1}\right)$ given by

$$
[\widehat{f}(\lambda) h](x)=\int_{\mathbb{R}^{n}} \mathfrak{F}^{2,3} f(x-y, \lambda(x+y) / 2, \lambda / 4) h(y) d y .
$$

where $\mathfrak{F}^{2,3}$ is the Fourier transform with respect to the second and the third variables on the Euclidean space $\mathbb{R}^{3}$. From $(1.4)$, the operator $\left(\widehat{\mu_{j, K}}(\lambda) \phi\right)(x)$ is given as $\int M_{j, K}^{\lambda}(x, \xi) \widehat{h}(\xi) d \xi$ where

$$
\begin{aligned}
M_{j, K}^{\lambda}(x, \xi) & =\int e^{-2 \pi i \Psi(\xi, y, \lambda, x)} \varphi_{j}(x-y) d y \\
\Psi(\xi, y, \lambda, x) & =\frac{\lambda 2^{k_{2}}(x-y)(x+y)}{2}+\frac{\lambda 2^{k_{3}}(x-y)}{4}-\xi y .
\end{aligned}
$$

The derivative of the above phase function $\Psi$ with respect to $y$ is given by

$$
\xi+\left(2^{k_{2}} \lambda y\right)+\left(\frac{2^{k_{3}} \lambda}{4}\right)
$$

It is convenient to regard $(\xi, \lambda y, \lambda)$ in $(1.6)$ as the three frequency variables. In the next section, we discuss the Littlewood-Paley type inequalities for the frequency variables $\xi, \lambda y, \lambda$ and their ratios. In Section $3, \mu_{j, K}$ is decomposed in the following three steps. The first step in the decomposition is to localize frequency variables $2^{k_{2}} \lambda y$, and $2^{k_{3}} \lambda$ in (1.6). Then we can split $\mu_{j, K}$ into the small frequency part $\mu_{j, K}^{l o c}$ and the large frequency part $\mu_{j, K}^{g l o}$. This is accomplished by 
the operators with convolution kernels $L_{j}^{\nu}$ 's below. Then the localized frequency maximal operator $\sup _{j, K} \mu_{j, K}^{l o c} * f$ is majorized by a variant of the strong maximal function on $\mathbb{H}^{1}$ in Section 4. Next step is to restrict $y$ in order to compare the size of two variables $\left(2^{k_{2}} \lambda y\right)$ and $\left(\frac{2^{k_{3}} \lambda}{4}\right)$. This is accomplished by the operators $A_{j}^{(2,3)}$ 's below. We split $\mu_{j, K}^{g l o}$ into $\left(2^{k_{2}} \lambda y\right)$ dominating part $m_{j, K}^{2}$ and $\left(\frac{2^{k_{3}} \lambda}{4}\right)$ dominating part $m_{j, K}^{3}$. The last step is to compare $\xi$ and the dominating term $\left(2^{k_{2}} \lambda y\right)$ or $\left(\frac{2^{k_{3}} \lambda}{4}\right)$. This is accomplished by operators $A_{j}^{(1,2)}$ or $A_{j}^{(1,3)}$. By this we split for each $\nu=2,3, m_{j, K}^{\nu}$ into the good decay part $m_{j, K}^{\nu, g}$ where (1.6) is kept away from zero and the bad decay part $m_{j, K}^{\nu, p}$ where (1.6) could be around zero. The good decay part $m_{j, K}^{\nu, g}$ is estimated by using the good decay property of $\Psi$ in (1.5) combined with the almost orthogonality Cotlar-Stein Lemma in Section 5 . The bad decay part $m_{j, K}^{\nu, p}$ is estimated in Section 5 by a version of the lacunary Littlewood-Paley decomposition. Here superscript $p$ represents Littlewood-Paley and $g$ represents good decay property.

Throughout this paper, the $L^{2}$ estimates for the convolution operators in $\mathbb{H}^{1}$ are made by applying the following proposition.

Proposition 1. Suppose that the operator $G$ is defined by $G f=k * f$ on $L^{2}\left(\mathbb{H}^{1}\right)$. Then

$$
\widehat{G f}(\lambda)=\widehat{k}(\lambda) \widehat{f}(\lambda)
$$

and the operator norm of $G$ on $L^{2}\left(\mathbb{H}^{1}\right)$ is given as the uniform $L^{2}\left(\mathbb{R}^{1}\right)$ norm of the Group Fourier transform of the convolution kernel $k$ in $\lambda$ :

$$
\|G\|_{L^{2}\left(\mathbb{H}^{1}\right) \mapsto L^{2}\left(\mathbb{H}^{1}\right)}=\frac{1}{2} \sup _{\lambda \in \mathbb{R}^{1}}\|\widehat{k}(\lambda)\|_{L^{2}\left(\mathbb{R}^{1}\right) \mapsto L^{2}\left(\mathbb{R}^{1}\right)} .
$$

See [5] and [10] for more details.

Notations : $\mathfrak{F}$ and $\mathfrak{F}^{-1}$ denotes the Fourier transform or its inverse for the given Euclidean space. Given two quantities $a$ and $b$, we write $a \lesssim b$ if there is a constant $C>0$ such that $a \leq C b$.

\section{Littlewood-Paley type inequalities}

2.1. Littlewood-Paley inequalities on $\mathbb{H}^{1}$. Let us choose an even function $\psi \in C_{0}^{\infty}(-1,1)$ such that $\psi \equiv 1$ on $[-1 / 2,1 / 2]$. Let $\chi(\xi)=\psi(\xi / 2)-\psi(\xi)$ and $\chi_{j}(\xi)=\chi\left(2^{j} \xi\right)$ for each integer $j$. Then $\chi_{j}$ is supported on $2^{-j-1} \leq|\xi| \leq 2^{-j+1}$ and $\sum_{j=-\infty}^{\infty} \chi_{j}(\xi) \equiv 1$. For $\nu=1,2,3$, we define a dyadic decomposition on each $\nu$-coordinate of $\mathbb{R}^{3}$ by using the measures:

$$
\begin{aligned}
L_{j}^{1}(y) & =\left[\mathfrak{F}^{-1} \chi_{j}\right]\left(y_{1}\right) \delta\left(y_{2}, y_{3}\right), \\
L_{j}^{2}(y) & =\left[\mathfrak{F}^{-1} \chi_{j}\right]\left(y_{2}\right) \delta\left(y_{1}, y_{3}\right), \\
L_{j}^{3}(y) & =\left[\mathfrak{F}^{-1} \chi_{j}\right]\left(y_{3}\right) \delta\left(y_{1}, y_{2}\right),
\end{aligned}
$$

where $\delta$ is a dirac mass on $\mathbb{R}^{2}$. Let us define $\Omega_{j}$ in $\mathbb{R}^{2}$ away from $\mathbb{R}^{1} \times\{0\}$ :

$$
\Omega_{j}(\xi, \eta)=\chi\left(\xi /\left(2^{j} \eta\right)\right) .
$$


Then $\Omega_{j}$ is supported in $\left\{2^{j-1} \leq|\xi / \eta| \leq 2^{j+1}\right\}$. We define a dyadic decomposition on the angular sectors by using the measures in $\mathbb{R}^{3}$ :

$$
\begin{aligned}
A_{j}^{(1,2)}(y) & =\left[\mathfrak{F}^{-1} \Omega_{j}\right]\left(y_{1}, y_{2}\right) \delta\left(y_{3}-2 y_{1} y_{2}\right), \\
A_{j}^{(1,3)}(y) & =\left[\mathfrak{F}^{-1} \Omega_{j}\right]\left(y_{1}, y_{3}\right) \delta\left(y_{2}\right), \\
A_{j}^{(2,3)}(y) & =\left[\mathfrak{F}^{-1} \Omega_{j}\right]\left(y_{2}, y_{3}\right) \delta\left(y_{1}\right),
\end{aligned}
$$

where $\delta$ is a dirac mass on $\mathbb{R}^{1}$. In [7], we have the following Littlewood-Paley type inequalities.

Lemma 1. For $\nu=1,2,3$, and $1<p<\infty$,

$$
\left\|\left(\sum_{j \in \mathbb{Z}}\left|L_{j}^{\nu} * f\right|^{2}\right)^{\frac{1}{2}}\right\|_{L^{p}\left(\mathbb{H}^{1}\right)} \lesssim\|f\|_{L^{p}\left(\mathbb{H}^{1}\right)} .
$$

Lemma 2. For $\omega=(1,2),(1,3),(2,3)$, and $1<p<\infty$,

$$
\left\|\left(\sum_{j \in \mathbb{Z}}\left|A_{j}^{\omega} * f\right|^{2}\right)^{\frac{1}{2}}\right\|_{L^{p}\left(\mathbb{H}^{1}\right)} \lesssim\|f\|_{L^{p}\left(\mathbb{H}^{1}\right)} .
$$

Lemma 1 and Lemma 2 for the case $\omega \neq(1,2)$ are prove by the following simple diffeomorphism invariance observation combined with the corresponding inequality on the Euclidean space. We may see this for the case $\omega=(1,3)$.

$A_{j}^{(1,3)} * f(x)=\int f_{x_{2}}\left(D_{x_{2}}\left(x_{1}-y_{1}, x_{3}-y_{3}-2 x_{1} x_{2}\right)\right) \mathfrak{F}^{-1}\left(\Omega_{j}\right)\left(y_{1}, y_{3}\right) d y_{1} d y_{3}$

where $f_{x_{2}}\left(x_{1}, x_{3}\right)=f\left(x_{1}, x_{2}, x_{3}\right)$ and $D_{x_{2}}\left(x_{1}, x_{3}\right)=\left(x_{1}, x_{3}+2 x_{1} x_{2}\right)$. By fixing $x_{2}$ and using the corresponding result on the Euclidean space, we obtain (2.2). The similar proof applies to the case $\omega=(2,3)$ and Lemma 1 . However for the proof of the case $\omega=(1,2)$, we need a multi-parameter Marcinkiewicz type multiplier theorem on the Heisenberg group. Note that for our $L^{p}$ estimate the product theory of the sub-Laplacian $\mathcal{L}=X^{2}+Y^{2}$ and $T$ in [8] can not be used. Here $X, Y, T$ are defined as $X=\frac{1}{2 \pi i}\left(\frac{\partial}{\partial p}+2 q \frac{\partial}{\partial t}\right)$ and $Y=\frac{1}{2 \pi i}\left(\frac{\partial}{\partial q}-2 p \frac{\partial}{\partial t}\right)$ and $T=\frac{1}{2 \pi i} \frac{\partial}{\partial t}$, which are three right invariant vector fields on the Heisenberg group $\mathbb{H}^{1}$. In proving the case $\omega=(1,2)$ in Lemma 2 , we need a product theory involving noncommutative vector fields $X$ and $Y$. This theory is developed by using the Kohn-Nirenberg functional calculus in [7].

For each $\nu=1,2,3$ and $\omega=(1,3),(2,3)$ let us define maximal functions:

$$
\mathcal{L}^{\nu} f=\sup _{j}\left[L_{j}^{\nu}\right]_{0} * f, \quad \mathcal{A}^{\omega} f=\sup _{j}\left[A_{j}^{\omega}\right]_{0} * f .
$$

where $\left[L_{j}^{\nu}\right]_{0}=\sum_{l=1}^{\infty} L_{j+l}^{\nu}$ and $\left[A_{j}^{\omega}\right]_{0}=\sum_{l=1}^{\infty} A_{j+l}^{\omega}$. Then for each $\nu=1,2,3$ and $\omega=(1,3),(2,3)$, the $L^{p}\left(\mathbb{H}^{1}\right)$ boundedness of $\mathcal{L}^{\nu}$ and $\mathcal{A}^{\omega}$ such that

$$
\left\|\mathcal{L}^{\nu} f\right\|_{L^{p}\left(\mathbb{H}^{1}\right)} \lesssim\|f\|_{L^{p}\left(\mathbb{H}^{1}\right)},\left\|\mathcal{A}^{\omega} f\right\|_{L^{p}\left(\mathbb{H}^{1}\right)} \lesssim\|f\|_{L^{p}\left(\mathbb{H}^{1}\right)}
$$

follows by using the above diffeomorphism and the corresponding Euclidean space result. In the same way we have the following vector valued inequalities. 
Lemma 3. Let $\nu=1,2,3$ and $\omega=(1,3),(2,3)$. Then we have for $1<p<\infty$,

$$
\begin{aligned}
\left(\sum_{j}\left|\mathcal{A}^{\omega} f_{j}\right|^{2}\right)^{\frac{1}{2}} \|_{L^{p}\left(\mathbb{H}^{1}\right)} & \lesssim\left\|\left(\sum_{j}\left|f_{j}\right|^{2}\right)^{\frac{1}{2}}\right\|_{L^{p}\left(\mathbb{H}^{1}\right)}, \\
\left(\sum_{j}\left|\mathcal{L}^{\nu} f_{j}\right|^{2}\right)^{\frac{1}{2}} \|_{L^{p}\left(\mathbb{H}^{1}\right)} & \lesssim\left\|\left(\sum_{j}\left|f_{j}\right|^{2}\right)^{\frac{1}{2}}\right\|_{L^{p}\left(\mathbb{H}^{1}\right)} .
\end{aligned}
$$

2.2. Directional maximal function with two parameters. For each fixed $k_{2}$ or $k_{3}$, we define the maximal function with two parameters:

$$
\begin{aligned}
& \mathcal{R}_{k_{2}}^{2} f=\sup _{j, k_{3}} \mu_{j, K} * f, \\
& \mathcal{R}_{k_{3}}^{3} f=\sup _{j, k_{2}} \mu_{j, K} * f .
\end{aligned}
$$

Lemma 4. For each $\nu=2,3$ with fixed $k \in \mathbb{Z},\left\|\mathcal{R}_{k}^{\nu} f\right\|_{L^{p}\left(\mathbb{H}^{1}\right)} \lesssim\|f\|_{L^{p}\left(\mathbb{H}^{1}\right)}$ with $1<p \leq \infty$.

A proof for Lemma 4 will be given in Section 6 .

Lemma 5. Suppose that (1.3) holds for some $p$. Then for $\frac{1}{q}<\frac{1}{2}\left(1+\frac{1}{p}\right)$, the following vector valued inequality holds for each fixed $\nu=2,3$

$$
\left\|\left(\sum_{k}\left|\mathcal{R}_{k}^{\nu} f_{k}\right|^{2}\right)^{1 / 2}\right\|_{L^{q}\left(\mathbb{H}^{1}\right)} \lesssim\left\|\left(\sum_{k}\left|f_{k}\right|^{2}\right)^{1 / 2}\right\|_{L^{q}\left(\mathbb{H}^{1}\right)}
$$

Proof. (2.6) follows from the interpolation of $L^{r}\left(l^{r}\left(\mathbb{H}^{1}\right)\right)$ with any $r>1$ and $L^{p}\left(l^{\infty}\left(\mathbb{H}^{1}\right)\right)$ with fixed $p$ for the vector valued operator $\left\{f_{k}\right\} \rightarrow\left\{\mathcal{R}_{k}^{\nu} f_{k}\right\}$.

\section{Decomposition}

In this section we decompose $\mu_{j, K}$ in three steps according to the size of the derivative of the phase function in (1.6). As the first step we localize $2^{j+k_{2}} \lambda y$ and $2^{j+k_{3}} \lambda$ in (1.6). Before doing this let us write the three operator $\widehat{L_{m}^{\nu}}(\lambda)$ by using computation formula (1.4):

$$
\begin{aligned}
\left(\widehat{L_{m}^{1}}(\lambda) h\right)(y) & =\iint e^{2 \pi i(y-z) \xi} \chi_{m}(\xi) d \xi h(z) d y, \\
\left(\widehat{L_{m}^{2}}(\lambda) h\right)(y) & =\chi_{m}(\lambda y) h(y), \\
\left(\widehat{L_{m}^{3}}(\lambda) h\right)(y) & =\chi_{m}(\lambda / 4) h(y) .
\end{aligned}
$$

We split $\mu_{j, K}=\mu_{j, K}^{l o c}+\mu_{j, K}^{g l}$ such that

$$
\begin{aligned}
& \mu_{j, K}^{l o c}=\mu_{j, K} *\left[L_{j+k_{2}}^{2}\right]_{0} *\left[L_{j+k_{3}}^{3}\right]_{0}, \\
& \mu_{j, K}^{g l o}=\mu_{j, K} *\left(\delta-\left[L_{j+k_{2}}^{2}\right]_{0} *\left[L_{j+k_{3}}^{3}\right]_{0}\right),
\end{aligned}
$$

where $\left[L_{j+k}^{\nu}\right]_{0}=\sum_{l=1}^{\infty} L_{j+k+l}^{\nu}$ with $\nu=2,3$. Then the maximal function $\sup _{j, K} \mu_{j, K}^{l o c} * f$ is handled by the majorization of a strong maximal function 
in Section 4. Before making the further decomposition it may be useful to know the three operators $\widehat{A_{m}^{\omega}}(\lambda)$ by computing (1.4):

$$
\begin{aligned}
& \left.\widehat{\left(A_{m}^{2,3}\right.}(\lambda) h\right)(y)=\Omega_{m}(\lambda y, \lambda) h(y), \\
& \left(\widehat{A_{m}^{1,2}}(\lambda) h\right)(y)=\int e^{2 \pi i \xi y} \Omega_{m}(\xi, \lambda y)[\mathfrak{F} h](\xi) d \xi, \\
& \left.\widehat{\left(A_{m}^{1,3}\right.}(\lambda) h\right)(y)=\int e^{2 \pi i \xi y} \Omega_{m}(\xi, \lambda)[\mathfrak{F} h](\xi) d \xi .
\end{aligned}
$$

As the second step, we need to compare two variables $2^{k_{2}} \lambda y$ and $\frac{2^{k_{3}} \lambda}{4}$ in (1.6). For this purpose let us define three measures by using $A_{m}^{2,3}$ in (3.2):

$$
\begin{aligned}
E_{k}^{1} & =\sum_{k-5 \leq m \leq k+5} A_{m}^{2,3}, \\
E_{k}^{2} & =\sum_{k+5<m} A_{m}^{2,3}, \\
E_{k}^{3} & =\sum_{m<k-5} A_{m}^{2,3} .
\end{aligned}
$$

Then let us decompose $\mu_{j, K}^{g l o}=m_{j, K}^{1}+m_{j, K}^{2}+m_{j, K}^{3}$ so that for each $\nu=1,2,3$,

$$
m_{j, K}^{\nu}=\mu_{j, K}^{g l o} * E_{k_{3}-k_{2}}^{\nu}
$$

The maximal function $\sup _{j, K} m_{j, K}^{1} * f$ is estimated in Section 6 by using the Littlewood-Paley inequality (2.2):

$$
\left\|\sup _{j, K} \mu_{j, K}^{g l o} * E_{k_{3}-k_{2}}^{1} * f\right\|_{L^{p}\left(\mathbb{H}^{1}\right)} \lesssim\|f\|_{L^{p}\left(\mathbb{H}^{1}\right)} .
$$

For $\nu=2,\left|2^{k_{2}} \lambda y\right| \gg\left|\frac{2^{k_{3}} \lambda}{4}\right|$ and that for $\nu=3,\left|2^{k_{2}} \lambda y\right| \ll\left|\frac{2^{k_{3}} \lambda}{4}\right|$ in (1.6). Note that the superscript 2 or 3 in (3.3) represents the dominating frequency variable between $\left|2^{k_{2}} \lambda y\right|$ and $\left|\frac{2^{k_{3}} \lambda}{4}\right|$.

As the third step, we compare $\xi$ and $2^{k_{2}} \lambda y$ for $\nu=2$ and compare $\xi$ and $\frac{2^{k_{3}} \lambda}{4}$ for $\nu=3$. For this purpose let us define for each $\nu=2,3$,

$$
\begin{aligned}
P_{k}^{\nu} & =\sum_{k-5 \leq m \leq k+5} A_{m}^{1, \nu}, \\
G_{k}^{\nu} & =\delta-P_{k}^{\nu} .
\end{aligned}
$$

Then we decompose that for each $\nu=2,3, m_{j, K}^{\nu}=m_{j, K}^{\nu, p}+m_{j, K}^{\nu, g}$ where

$$
\begin{aligned}
& m_{j, K}^{\nu, p}=m_{j, K}^{\nu} * P_{k_{\nu}}^{\nu}, \\
& m_{j, K}^{\nu, g}=m_{j, K}^{\nu} * G_{k_{\nu}}^{\nu} .
\end{aligned}
$$

The maximal operator $\sup _{j, K} m_{j, K}^{\nu, p} * f$ is estimated in Section 5 by using the Littlewood-Paley inequality (2.2). Here the subscript $p$ indicates "LittlewoodPaley". 
Let us rewrite $m_{j, K}^{\nu, g}=\mu_{j, K} *\left(\delta-\left[L_{j+k_{2}}^{2}\right]_{0} *\left[L_{j+k_{3}}^{3}\right]_{0}\right) * E_{k_{3}-k_{2}}^{\nu} * G_{k_{\nu}}^{\nu}$. Set two functions $\theta_{j, K}^{2}(x, y, \lambda)$ and $\theta_{j, K}^{3}(x, y, \lambda)$ such that each of which is given by

$$
\begin{aligned}
& \varphi_{j}(x-y)\left(1-\psi\left(2^{j+k_{2}} \lambda y\right) \psi\left(2^{j+k_{3}} \lambda\right)\right)\left(1-\psi\left(\frac{2^{k_{2}} \lambda y}{2^{k_{3}+6} \lambda}\right)\right), \\
& \varphi_{j}(x-y)\left(1-\psi\left(2^{j+k_{2}} \lambda y\right) \psi\left(2^{j+k_{3}} \lambda\right)\right) \psi\left(\frac{2^{k_{2}} \lambda y}{2^{k_{3}-5} \lambda}\right) .
\end{aligned}
$$

Then from $(1.7),(3.1),(3.2),(3.7)$ and (3.8), the integral kernel $\mathcal{K}_{j, K}^{\nu}(x, \xi)$ of the operator $\widehat{m_{j, K}^{\nu, g}}(\lambda) \mathfrak{F}^{-1}$ with each $\nu=2,3$ is

$$
\begin{aligned}
\mathcal{K}_{j, K}^{2}(x, \xi) & =\int e^{-2 \pi i \Psi(\xi, y, \lambda, x)} \theta_{j, K}^{2}(x, y, \lambda)\left(1-\sum_{-5 \leq m \leq 5} \Omega_{k_{2}+m}(\xi, \lambda y)\right) d y, \\
\mathcal{K}_{j, K}^{3}(x, \xi) & =\int e^{-2 \pi i \Psi(\xi, y, \lambda, x)} \theta_{j, K}^{3}(x, y, \lambda)\left(1-\sum_{-5 \leq m \leq 5} \Omega_{k_{3}+m}(\xi, \lambda)\right) d y .
\end{aligned}
$$

We see that the support of the above integrand is contained in the set $B(\nu)$ :

$$
\begin{aligned}
& B(2)=\left(\left\{|\xi| \gg\left|2^{k_{2}} \lambda y\right|\right\} \cup\left\{|\xi| \ll\left|2^{k_{2}} \lambda y\right|\right\}\right) \cap\left\{\left|2^{k_{2}} \lambda y\right| \gg\left|\frac{2^{k_{3}} \lambda}{4}\right|\right\}, \\
& B(3)=\left(\left\{|\xi| \gg\left|2^{k_{3}} \lambda\right|\right\} \cup\left\{|\xi| \ll\left|2^{k_{3}} \lambda\right|\right\}\right) \cap\left\{\left|2^{k_{2}} \lambda y\right| \ll\left|\frac{2^{k_{3}} \lambda}{4}\right|\right\} .
\end{aligned}
$$

On each $B(\nu)$ the derivative of phase function $\Psi_{y}^{\prime}$ is kept away from zero. This is the good part. The maximal operator $\sup _{j, K} m_{j, K}^{\nu, g} * f$ is estimated in Section 5 by using the good decay property of the phase function in (1.6) combined with the almost orthogonality lemma. Note the superscript $g$ indicates "good decay". In the next two sections we prove the following five estimates with $\nu=2,3$ for the proof of Theorem 1 .

$$
\begin{gathered}
\left\|\sup _{j, K} \mu_{j, K}^{l o c} * f\right\|_{L^{p}\left(\mathbb{H}^{1}\right)} \lesssim\|f\|_{L^{p}\left(\mathbb{H}^{1}\right)}, \\
\left\|\sup _{j, K} m_{j, K}^{\nu, p} * f\right\|_{L^{p}\left(\mathbb{H}^{1}\right)} \lesssim\|f\|_{L^{p}\left(\mathbb{H}^{1}\right)}, \\
\left\|\sup _{j, K} m_{j, K}^{\nu, g} * f\right\|_{L^{p}\left(\mathbb{H}^{1}\right)} \lesssim\|f\|_{L^{p}\left(\mathbb{H}^{1}\right)} .
\end{gathered}
$$

\section{Majorization by a variant of strong maximal function}

In this section we show (3.9). Let $\widehat{\psi}_{j}(u)=\widehat{\psi}\left(u / 2^{j}\right) / 2^{j}$. Then for $y \in \mathbb{H}^{1}$ we can compute $\mu_{j, K}^{l o c}(y)=\mu_{j, K} *\left[L_{j+k_{2}}^{2}\right]_{0} *\left[L_{j+k_{3}}^{3}\right]_{0}(y)$ :

$$
\mu_{j, K}^{l o c}(y)=\varphi_{j}\left(y_{1}\right) \widehat{\psi}_{j+k_{2}}\left(y_{2}-2^{k_{2}} y_{1}\right) \widehat{\psi}_{j+k_{3}}\left(y_{3}-2\left(y_{2}-2^{k_{2}} y_{1}\right) y_{1}-2^{k_{3}} y_{1}\right) .
$$

Let $j=j_{1}, j+k_{2}=j_{2}, j+k_{3}=j_{3}$, then $\mu_{j, K}^{l o c}(y)$ is majorized by the function

$$
\Phi_{j_{1}, j_{2}}^{(1,2)} * \Phi_{j_{3}}^{3}(y)
$$


where for $\phi_{j}(u)=\phi\left(u / 2^{j}\right) / 2^{j}$ with some $\phi$ in the Schwartz class $\mathcal{S}(\mathbb{R})$,

$$
\begin{aligned}
\Phi_{j_{1}, j_{2}}^{(1,2)}(y) & =\phi_{j_{1}}\left(y_{1}\right) \phi_{j_{2}}\left(y_{2}\right) \delta\left(y_{3}-2\left(y_{2}-2^{-j_{1}+j_{2}} y_{1}\right) y_{1}\right), \\
\Phi_{j_{3}}^{3}(y) & =\delta\left(y_{1}, y_{2}\right) \phi_{j_{3}}\left(y_{3}\right) .
\end{aligned}
$$

Therefore for all $x \in \mathbb{H}^{1}$,

$$
\left|\sup _{j, K} \mu_{j, K}^{l o c} * f(x)\right| \leq\left|\sup _{j_{1}, j_{2}, j_{3}} \Phi_{j_{1}, j_{2}}^{(1,2)} * \Phi_{j_{3}}^{3} * f(x)\right| .
$$

Thus (3.9) is proved from the $L^{p}\left(\mathbb{H}^{1}\right)$ boundedness of the maximal function $\sup _{l} \Phi_{l}^{3} * f$ (which follows from the boundedness of the one dimensional HardyLittlewood maximal function) and Lemma 6 below.

Lemma 6. For $1<p \leq \infty$, we have $\left\|\sup _{J} \Phi_{J}^{(1,2)} * f\right\|_{L^{p}\left(\mathbb{H}^{1}\right)} \lesssim\|f\|_{L^{p}\left(\mathbb{H}^{1}\right)}$, where $J=\left(j_{1}, j_{2}\right) \in \mathbb{Z}^{2}$.

The above maximal operator is one variant of the standard strong maximal function defined by

$$
\sup _{r_{1}, r_{2}, r_{3}>0} \frac{1}{2^{3} r_{1} r_{2} r_{3}} \int_{-r_{1}}^{r_{1}} \int_{-r_{2}}^{r_{2}} \int_{-r_{3}}^{r_{3}} f\left(x \cdot y^{-1}\right) d y .
$$

The $L^{p}\left(\mathbb{H}^{1}\right)$ boundedness of this strong maximal function and its generalization on the Nilpotent group are obtained in [3].

Let us prove Lemma 6 . The basic ideas are similar to the one in [3], but there will be some technical difficulties. Let $\Phi_{j}^{1}(y)=\delta\left(y_{2}, y_{3}\right) \phi_{j}\left(y_{1}\right)$ and $\Phi_{j}^{2}(y)=$ $\delta\left(y_{1}, y_{3}\right) \phi_{j}\left(y_{2}\right)$. Since we have $\left\|\sup _{J} \Phi_{j_{1}}^{1} * \Phi_{j_{2}}^{2} * f\right\|_{L^{p}\left(\mathbb{H}^{1}\right)} \lesssim f \|_{L^{p}\left(\mathbb{H}^{1}\right)}$, it suffices to show that

$$
\left\|\sup _{J} M_{J} * f\right\|_{L^{p}\left(\mathbb{H}^{1}\right)} \lesssim\|f\|_{L^{p}\left(\mathbb{H}^{1}\right)} .
$$

where $M_{J}=\Phi_{J}^{(1,2)}-\Phi_{j_{1}}^{1} * \Phi_{j_{2}}^{2}$. Let $L_{J}=L_{j_{2}}^{2} * L_{j_{1}}^{1}$ and its adjoint $L_{J}^{*}=L_{j_{1}}^{1} * L_{j_{2}}^{2}$. Then (4.1) follows from the two estimates: For $a, b \in \mathbb{Z}^{2}$,

$$
\begin{aligned}
& \left\|\left(\sum_{J}\left|L_{J+b}^{*} * M_{J} * L_{J+a} * f\right|^{2}\right)^{1 / 2}\right\|_{L^{p}\left(\mathbb{H}^{1}\right)} \lesssim\|f\|_{L^{p}\left(\mathbb{H}^{1}\right)}, \\
& \left\|\left(\sum_{J}\left|L_{J+b}^{*} * M_{J} * L_{J+a} * f\right|^{2}\right)^{1 / 2}\right\|_{L^{2}\left(\mathbb{H}^{1}\right)} \lesssim 2^{-c(|a|+|b|)}\|f\|_{L^{2}\left(\mathbb{H}^{1}\right)} .
\end{aligned}
$$

Proof of (4.2). Assume that (4.3) is true. Then (4.1) holds for $p=2$. Let us consider the vector valued operator $\left\{f_{J}\right\} \mapsto\left\{M_{J} * f_{J}\right\}$. By the interpolation of $L^{2}\left(l^{\infty}\right)$ and $L^{1}\left(l^{1}\right)$, we have for $\frac{4}{3}<p \leq 2$ :

$$
\left\|\left(\sum_{J}\left|M_{J} * f_{J}\right|^{2}\right)^{\frac{1}{2}}\right\|_{L^{p}\left(\mathbb{H}^{1}\right)} \lesssim\left\|\left(\sum_{J}\left|f_{J}\right|^{2}\right)^{\frac{1}{2}}\right\|_{L^{p}\left(\mathbb{H}^{1}\right)}
$$

Thus (4.2) follows from (2.1) and (4.4). A standard bootstrap argument extends this range to $1<p \leq 2$. 
Let us prove (4.3). From the duality combined with Proposition 1, it suffices to show that for $J=\left(j_{1}, j_{2}\right)$ and $J^{\prime}=\left(j_{1}^{\prime}, j_{2}^{\prime}\right)$

$$
\begin{gathered}
\left\|\widehat{M_{J}}(\lambda) \widehat{L_{J+a}}(\lambda)\right\|_{o p} \lesssim 2^{-c|a|} \\
\left\|\widehat{L_{J}}(\lambda) \widehat{L_{J^{\prime}}^{*}}(\lambda)\right\|_{o p} \lesssim 2^{-c\left|J-J^{\prime}\right|}
\end{gathered}
$$

Proof of (4.6). By using (1.4),(1.7) and (3.1),

$$
\left(\widehat{L_{J}}(\lambda) h\right)(x)=\int \chi_{j_{2}}(\lambda x) \chi_{j_{1}}(\xi) e^{2 \pi i \xi x}(\mathfrak{F} h)(\xi) d \xi .
$$

Thus the integral kernel of the operator $\widehat{L_{J}}(\lambda) \mathfrak{F}^{-1}$ is given as

$$
L_{J}(x, \xi)=\chi_{j_{2}}(\lambda x) \chi_{j_{1}}(\xi) e^{2 \pi i \xi x} .
$$

So the kernel $L_{J, J^{\prime}}(x, y)$ for the composition operator $\widehat{L_{J}}(\lambda) \widehat{L_{J^{\prime}}^{*}}(\lambda)$ is

$$
L_{J, J^{\prime}}(x, y)=\int e^{2 \pi i \xi(x-y)} \chi_{j_{2}}(\lambda x) \chi_{j_{1}}(\xi) \chi_{j_{2}^{\prime}}(\lambda y) \chi_{j_{1}^{\prime}}(\xi) d \xi
$$

From the support condition for $\chi_{j_{1}}$ and $\chi_{j_{1}^{\prime}}$, we have $\left|j_{1}-j_{1}^{\prime}\right|<3$. Next we apply the integration by parts with respect to $d \xi$ on the above integral to obtain the desired $L^{1}$ norm $2^{-\left|j_{2}-j_{2}^{\prime}\right|}$ for the kernel $L_{J, J^{\prime}}(x, y)$ with one fixed variable $x$ or $y$.

Let us show (4.5). From (1.4) we compute the integral kernel $M_{J}^{\lambda}(x, y)$ for $\widehat{M_{J}}(\lambda)$ :

$$
M_{J}^{\lambda}(x, y)=\phi_{j_{1}}(x-y)\left(e^{2 \pi i \lambda 2^{\left(j_{2}-j_{1}\right)}(x-y)^{2}}-1\right)(\mathfrak{F} \phi)\left(2^{j_{2}} \lambda y\right) .
$$

Let us fix $\lambda \approx 2^{-j_{3}}$ and decompose $\widehat{M_{J}}(\lambda) \widehat{L_{J+a}}(\lambda)=\sum_{k \in \mathbb{Z}} \mathcal{M}_{k, J}$ where

$$
\begin{aligned}
& \mathcal{M}_{k, J} f(x)=\int M_{k, J}(x, y) f(y) d y, \\
& M_{k, J}(x, y)=\chi\left(\frac{x-y}{2^{k-j_{2}+j_{3}}}\right) \phi_{j_{1}}(x-y)\left(e^{2 \pi i \lambda 2^{\left(j_{2}-j_{1}\right)}(x-y)^{2}}-1\right)(\mathfrak{F} \phi)\left(2^{j_{2}} \lambda y\right) .
\end{aligned}
$$

For the proof of (4.5), we show the uniform estimates in $k, J$ and $\lambda$ :

$$
\begin{aligned}
& \left\|\mathcal{M}_{k, J}\right\|_{o p} \lesssim 2^{-c|k|} \\
& \left\|\mathcal{M}_{k, J} \widehat{L_{J+a}}(\lambda)\right\|_{o p} \lesssim 2^{-c|a|} .
\end{aligned}
$$

Proof of (4.7). Using the decay for $\phi$ and the mean value property for the exponential function, we have

$$
\begin{aligned}
& \left|M_{k, J}(x, y)\right| \lesssim \frac{\chi\left(\frac{x-y}{2^{k-j_{2}+j_{3}}}\right)\left|(\mathfrak{F} \phi)\left(2^{j_{2}} \lambda y\right)\right|}{|x-y|\left(1+\left|2^{-j_{1}}(x-y)\right|\right)^{N}} \\
& \left|\phi_{j_{1}}(x-y)\left(e^{2 \pi i \lambda 2^{\left(j_{2}-j_{1}\right)}(x-y)^{2}}-1\right)\right| \lesssim \frac{2^{-j_{1}}\left|\lambda(x-y) 2^{j_{2}}\right|}{\left(1+\left|2^{-j_{1}}(x-y)\right|\right)^{N}} .
\end{aligned}
$$


When $k \geq 0$, by using (4.9) we have the $L^{1}$ norm with each fixed $x$ or $y$,

$$
\begin{aligned}
& \int\left|M_{k, J}(x, y)\right| d y \lesssim 2^{-k}, \\
& \int\left|M_{k, J}(x, y)\right| d x \lesssim 1 .
\end{aligned}
$$

When $k<0$, we apply (4.10) to obtain that for each fixed $x$ or $y$,

$$
\begin{aligned}
& \int\left|M_{k, J}(x, y)\right| d y \lesssim 2^{k}, \\
& \int\left|M_{k, J}(x, y)\right| d x \lesssim 1 .
\end{aligned}
$$

(4.7) follows from (4.11) and (4.12).

Proof of (4.8). For the estimate of $\left\|\mathcal{M}_{k, J} \widehat{L_{j_{2}+a_{2}}^{2}}(\lambda) \widehat{L_{j_{1}+a_{1}}^{1}}(\lambda)\right\|_{o p}$, let us first show that for some $c>0$,

$$
\left\|\mathcal{M}_{k, J} \widehat{L_{j_{2}+a_{2}}^{2}}(\lambda)\right\|_{o p} \lesssim 2^{-c\left|a_{2}\right|} .
$$

Since we have (4.7), it suffices to consider the case $\left|a_{2}\right| \gg|k|$. The integral kernel of $\mathcal{M}_{k, J} \widehat{L_{j_{2}+a_{2}}^{2}}(\lambda)$ is given by $M_{k, J}(x, y) \chi_{j_{2}+a_{2}}(\lambda y)$.

When $a_{2} \geq 0$ : from (4.9),

$$
\begin{gathered}
\int\left|M_{k, J}(x, y) \chi_{j_{2}+a_{2}}(\lambda y)\right| d y \lesssim \frac{2^{-a_{2}-j_{2}+j_{3}}}{2^{k-j_{2}+j_{3}}} \lesssim 2^{-c a_{2}}, \\
\int\left|M_{k, J}(x, y) \chi_{j_{2}+a_{2}}(\lambda y)\right| d x \lesssim 1
\end{gathered}
$$

When $a_{2}<0$ : from the support condition for $\chi_{j_{2}+a_{2}}(\lambda y)$, we have

$$
\left|(\mathfrak{F} \phi)\left(2^{j_{2}} \lambda y\right)\right| \leq 2^{-\left|a_{2}\right|} .
$$

Therefore

$$
\begin{gathered}
\int\left|M_{k, J}(x, y) \chi_{j_{2}+a_{2}}(\lambda y)\right| d y \lesssim 2^{-c\left|a_{2}\right|} \\
\int\left|M_{k, J}(x, y) \chi_{j_{2}+a_{2}}(\lambda y)\right| d x \lesssim 2^{-c\left|a_{2}\right|} .
\end{gathered}
$$

Hence (4.14) and (4.15) prove (4.13). Let us complete the proof of (4.8). From (4.7) we may assume that $\left|a_{1}\right| \gg|k|$. Using (1.4) we write the integral kernel $M_{k, J}^{a}(x, \eta)$ of $\mathcal{M}_{k, J} \widehat{L_{J+a}}(\lambda) \mathfrak{F}^{-1}$ :

$$
M_{k, J}^{a}(x, \eta)=\int e^{2 \pi i \eta y} M_{k, J}(x, y) \chi_{j_{2}+a_{2}}(\lambda y) \chi_{j_{1}+a_{1}}(\eta) d y
$$


When $a_{1}<0$, by using integration by parts for the above integral we have the $L^{1}$ norm estimate of $M_{k, J}^{a}$ for each fixed $x$ or $\eta$,

$$
\begin{aligned}
& \iint\left|\frac{\chi_{j_{1}+a_{1}}(\eta)}{2 \pi i \eta} \frac{\partial}{\partial y}\left(M_{k, J}(x, y) \chi_{j_{2}+a_{2}}(\lambda y)\right)\right| d y d \eta \lesssim 2^{-j_{1}}, \\
& \iint\left|\frac{\chi_{j_{1}+a_{1}}(\eta)}{2 \pi i \eta} \frac{\partial}{\partial y}\left(M_{k, J}(x, y) \chi_{j_{2}+a_{2}}(\lambda y)\right)\right| d y d x \lesssim 2^{j_{1}+a_{1}} .
\end{aligned}
$$

Here we used (4.10) and $\left|\phi\left(2^{-j_{1}}(x-y)\right) \partial_{y}\left(e^{i \lambda 2^{\left(j_{2}-j_{1}\right)}(x-y)^{2}}\right)\right| \leq\left|\lambda 2^{j_{2}}\right|$ for the above computation. When $a_{1}>0$, we have from (4.9) that

$$
\begin{aligned}
& \iint\left|\chi_{j_{1}+a_{1}}(\eta) M_{k, J}(x, y) \chi_{j_{2}+a_{2}}(\lambda y)\right| d y d \eta \lesssim 2^{-j_{1}-a_{1}}, \\
& \iint\left|\chi_{j_{1}+a_{1}}(\eta) M_{k, J}(x, y) \chi_{j_{2}+a_{2}}(\lambda y)\right| d y d x \lesssim 2^{j_{1}+|k|} .
\end{aligned}
$$

Hence (4.8) follows from (4.16) and (4.17) combined with (4.13).

\section{Littewood-Paley inequality and almost orthogonality}

In this section we prove (3.10) and (3.11). Recall that for each $\nu=2,3$,

$$
m_{j, K}^{\nu, p}=\mu_{j, K} *\left(\delta-\left[L_{j+k_{2}}^{2}\right]_{0} *\left[L_{j+k_{3}}^{3}\right]_{0}\right) * E_{k_{3}-k_{2}}^{\nu} * P_{k_{\nu}}^{\nu} .
$$

Proof of (3.10). From Lemma 2, Lemma 3 and Lemma 5, we have for each $\nu=2,3$ and $1<p<\infty$ :

$$
\begin{aligned}
\left\|\sup _{j, K} m_{j, K}^{\nu, p} * f\right\|_{L^{p}\left(\mathbb{H}^{1}\right)} & \lesssim\left\|\left(\sum_{k}\left|\mathcal{R}_{k}^{\nu} \mathcal{L}^{2} \mathcal{L}^{3} \mathcal{A}^{23}\left(P_{k}^{\nu} * f\right)\right|^{2}\right)^{\frac{1}{2}}\right\|_{L^{p}\left(\mathbb{H}^{1}\right)} \\
& +\left\|\left(\sum_{k}\left|\mathcal{R}_{k}^{\nu} \mathcal{A}^{23}\left(P_{k}^{\nu} * f\right)\right|^{2}\right)^{\frac{1}{2}}\right\|_{L^{p}\left(\mathbb{H}^{1}\right)} \\
& \lesssim\|f\|_{L^{p}\left(\mathbb{H}^{1}\right)} .
\end{aligned}
$$

This proves (3.10).

So there remains the case (3.11). Let us define two measures:

$$
\begin{aligned}
& \mu_{j, k}^{2}(f)=\int f\left(\left(1,2^{k}, 0\right) t\right) \varphi_{j}(t) d t \\
& \mu_{j, k}^{3}(f)=\int f\left(\left(1,0,2^{k}\right) t\right) \varphi_{j}(t) d t .
\end{aligned}
$$

With these measures, set

$$
\begin{aligned}
& n_{j, K}^{2}=\mu_{j, k_{2}}^{2} *\left(\delta-\left[L_{j+k_{2}}^{2}\right]_{0} *\left[L_{j+k_{3}}^{3}\right]_{0}\right) * E_{k_{3}-k_{2}}^{2}, \\
& n_{j, K}^{3}=\mu_{j, k_{3}}^{3} *\left(\delta-\left[L_{j+k_{2}}^{2}\right]_{0} *\left[L_{j+k_{3}}^{3}\right]_{0}\right) * E_{k_{3}-k_{2}}^{3} .
\end{aligned}
$$

From Lemma 4 and (2.3),

$$
\left\|\sup _{j, K} n_{j, K}^{\nu} * f\right\|_{L^{p}\left(\mathbb{H}^{1}\right)} \lesssim\|f\|_{L^{p}\left(\mathbb{H}^{1}\right)}
$$


For $L=\left(l_{1}, l_{2}, l_{3}\right)$, we put

$$
\begin{aligned}
& \mathcal{L}_{j, K, L}^{2}=L_{j+k_{3}-l_{3}}^{3} * L_{j+k_{2}-l_{2}}^{2} * G_{k_{2}}^{2} * L_{j-l_{1}}^{1} \\
& \mathcal{L}_{j, K, L}^{3}=L_{j+k_{3}-l_{3}}^{3} * L_{j+k_{2}-l_{2}}^{2} * G_{k_{3}}^{3} * L_{j-l_{1}}^{1} .
\end{aligned}
$$

We can see that $\sum_{L} \mathcal{L}_{j, K, L}^{\nu}=G_{k_{\nu}}^{\nu}$. So for the proof of (3.11) it suffices to show that for $\mathcal{N}_{j, K}^{\nu}=\left(m_{j, K}^{\nu}-n_{j, K}^{\nu}\right)$ with $\nu=2,3$, and $1<p<\infty$,

$$
\begin{aligned}
\left\|\left(\sum_{j, K}\left|\mathcal{N}_{j, K}^{\nu} * \mathcal{L}_{j, K, L}^{\nu} * f\right|^{2}\right)^{1 / 2}\right\|_{L^{p}\left(\mathbb{H}^{1}\right)} & \lesssim\|f\|_{L^{p}\left(\mathbb{H}^{1}\right)} \\
\left\|\left(\sum_{j, K}\left|\mathcal{N}_{j, K}^{\nu} * \mathcal{L}_{j, K, L}^{\nu} * f\right|^{2}\right)^{1 / 2}\right\|_{L^{2}\left(\mathbb{H}^{1}\right)} & \lesssim 2^{-c|L|}\|f\|_{L^{2}\left(\mathbb{H}^{1}\right)} .
\end{aligned}
$$

Proof of (5.2). Assume that (5.3) holds. Then (5.2) for $4 / 3<p \leq 2$ follows from Lemma 1,2 and the interpolation of vector valued operator $\left\{f_{j, K}\right\} \rightarrow\left\{\mathcal{N}_{j, K}^{\nu} *\right.$ $\left.f_{j, K}\right\}$. A bootstrap argument gives full range of $p>1$.

Proof of (5.3). From the duality and Proposition 1, we have only to show that for $\nu=2,3$,

$$
\begin{aligned}
\left\|\widehat{\mathcal{L}_{j, K, L}^{\nu}}(\lambda)\left[\widehat{\mathcal{L}_{j^{\prime}, K^{\prime}, L}}(\lambda)\right]^{*}\right\|_{o p} & \lesssim 2^{-c\left(\left|j-j^{\prime}\right|+\left|K-K^{\prime}\right|\right)}, \\
\left\|\widehat{\mathcal{N}_{j, K}^{\nu}}(\lambda) \widehat{\mathcal{L}_{j, K, L}^{\nu}}(\lambda)\right\|_{o p} & \lesssim 2^{-c|L|} .
\end{aligned}
$$

(5.4) is obtained by applying the same proof of (4.6). For this we use the fact that $L_{j_{1}}^{3} * L_{j_{2}}^{3}=0$ with $\left|j_{1}-j_{2}\right|>2$ and that $L_{j}^{3}$ is commutative with any other $L_{j}^{\nu}$ and $A_{j}^{\omega}$ 's. Let us prove (5.5). We compute the integral kernel for $\widehat{\mathcal{N}_{j, K}^{\nu}}(\lambda) \widehat{\mathcal{L}_{j, K, L}^{\nu}}(\lambda)$ :

$$
N_{j, K, L}^{\nu}(x, \xi)=\int \Psi_{K}^{\nu}(x, y) V_{j, K, L}^{\nu}(x, y, \xi) d y .
$$

Here the oscillatory part is given by

$$
\begin{aligned}
& \Psi_{K}^{2}(x, y)=\left(e^{i \lambda\left[2^{k_{2}}(x-y)(x+y)+2^{k_{3}}(x-y) / 4\right]}-e^{i \lambda 2^{k_{2}}(x-y)(x+y) / 2}\right) e^{i \xi y}, \\
& \Psi_{K}^{3}(x, y)=\left(e^{i \lambda\left[2^{k_{2}}(x-y)(x+y)+2^{k_{3}}(x-y) / 4\right]}-e^{i \lambda 2^{k_{3}}(x-y) / 4}\right) e^{i \xi y} .
\end{aligned}
$$

where we omit $2 \pi$ for simplicity. The amplitude parts

$$
V_{j, K, L}^{2}(x, y, \xi) \text { and } V_{j, K, L}^{3}(x, y, \xi)
$$

are given by

$$
\begin{aligned}
& \theta_{j, K}^{2}(x, y, \lambda) \chi_{j+k_{2}-l_{2}}(\lambda y) \chi_{j+k_{3}-l_{3}}(\lambda) \chi_{j-l_{1}}(\xi)\left(1-\sum_{-5 \leq m \leq 5} \Omega_{k_{2}+m}(\xi, \lambda y)\right) \\
& \theta_{j, K}^{3}(x, y, \lambda) \chi_{j+k_{2}-l_{2}}(\lambda y) \chi_{j+k_{3}-l_{3}}(\lambda) \chi_{j-l_{1}}(\xi)\left(1-\sum_{-5 \leq m \leq 5} \Omega_{k_{3}+m}(\xi, \lambda)\right) .
\end{aligned}
$$


where $\theta^{2}$ and $\theta^{3}$ are defined in (3.7) and (3.8). Note that on the support of $V_{j, K, L}^{2}(x, y, \xi), l_{2}>0$ and $l_{2} \geq l_{3}$, and on the support of $V_{j, K, L}^{3}(x, y, \xi), l_{3}>0$ and $l_{3} \geq l_{2}$. We apply the mean value property for (5.7) to obtain that

$$
\begin{gathered}
\left\|\widehat{\mathcal{N}_{j, K}^{2}}(\lambda) \widehat{L_{j+k_{3}-l_{3}}^{3}}(\lambda)\right\|_{o p} \lesssim \min \left(1,2^{l_{3}}\right) \\
\left\|\widehat{\mathcal{N}_{j, K}^{3}}(\lambda) L_{j+k_{2}-l_{2}}^{2}(\lambda)\right\|_{o p} \lesssim \min \left(1,2^{l_{2}}\right)
\end{gathered}
$$

On the support of $V_{j, K, L}^{\nu}(x, y, \xi)$, the derivative of each phase function in (5.6) is bounded below from $\left(|\xi|+\left|2^{k_{2}} \lambda y\right|+\left|2^{k_{3}} \lambda\right|\right)$. Thus by using this we apply integration by parts for the $y$ variable in (5.6) to obtain for each $\nu=2,3$,

$$
\begin{aligned}
\int\left|N_{j, K, L}^{\nu}(x, \xi)\right| d x & \lesssim \frac{2^{j}}{2^{l_{\nu}}+2^{l_{1}}}, \\
\int\left|N_{j, K, L}^{\nu}(x, \xi)\right| d \xi & \lesssim \frac{2^{-j} 2^{l_{1}}}{2^{l_{\nu}}+2^{l_{1}}} .
\end{aligned}
$$

By (5.9), we have $\left\|\widehat{\mathcal{N}_{j, K}^{\nu}}(\lambda) \widehat{\mathcal{L}_{j, K, L}^{\nu}}(\lambda)\right\|_{o p} \lesssim 2^{-\left(\left|l_{1}\right|+\left|l_{\nu}\right|\right) / 4}$ for each $\nu=2,3$. This and (5.8) give us the desired estimate for (5.5). So we are done with the proof of (5.3).

Therefore our five estimates in (3.9)-(3.11) are finished.

\section{Proofs of Lemma 4 and (3.4)}

Proof for $\mathcal{R}_{k_{2}}^{2}$. Let us define two diffeomorphisms $D_{1,2}^{a}\left(y_{1}, y_{2}, y_{3}\right)=\left(y_{1}, y_{2}+\right.$ $\left.2^{a} y_{1}, y_{3}\right)$ and $D\left(y_{1}, y_{2}, y_{3}\right)=\left(y_{1}, y_{2}, y_{3}-2 y_{1} y_{2}\right)$. Then $\mu_{j, K} f(x)$ is rewritten as

$$
\int f\left(D_{1,2}^{k_{2}} D\left(x_{1}-t, x_{2}-2^{k_{2}} x_{1}, x_{3}-2^{k_{3}} t+2 x_{1}\left(x_{2}-2^{k_{2}} x_{1}\right)\right) \varphi_{j}(t) d t .\right.
$$

Thus the $L^{p}\left(\mathbb{H}^{1}\right)$ boundedness of $\mathcal{R}_{k_{2}}^{2}$ follows from the $L^{p}\left(\mathbb{R}^{2}\right)$ boundedness of the lacunary directional maximal function $M_{2}^{\text {lac }}$ in (1.1).

Proof for $\mathcal{R}_{k_{3}}^{3}$. It suffices to consider the maximal function $\sup _{j, k_{2}} \mu_{j, k}^{2} * f$ where $\mu_{j, k}^{2}$ is defined in (5.1). We split $\mu_{j, k_{2}}=\mu_{j, k_{2}}^{l o c}+\mu_{j, k_{2}}^{g l o}$ such that $\mu_{j, k_{2}}^{l o c}=\mu_{j, k_{2}} *$ $\left[L_{j+k_{2}}^{2}\right]_{0}$ and $\mu_{j, k_{2}}^{g l o}=\mu_{j, K} *\left(\delta-\left[L_{j+k_{2}}^{2}\right]_{0}\right)$. Then $\mu_{j, k_{2}}^{\text {loc }}$ is handled by Lemma 6 and the fact

$$
\sup _{j, k_{2}}\left|\mu_{j, k_{2}}^{l o c} * f(x)\right| \lesssim \sup _{J \in \mathbb{Z}^{2}}\left|\Phi_{J}^{(1,2)} * f(x)\right| .
$$

Let $\mu_{j, k_{2}}^{g l o}=\mu_{j, k_{2}}^{g l o} * P_{k_{2}}^{2}+\mu_{j, k_{2}}^{g l o} * G_{k_{2}}^{2}$. By the $L^{p}$ boundedness of the maximal function $\sup _{j} \mu_{j, k_{2}} * f$ and Lemma 1,3 ,

$$
\begin{aligned}
\left\|\left(\sum_{k_{2}}\left|\sup _{j} \mu_{j, k_{2}}^{g l o} * P_{k_{2}}^{2} * f\right|^{2}\right)^{\frac{1}{2}}\right\|_{L^{p}\left(\mathbb{H}^{1}\right)} & \lesssim\left\|\left(\sum_{k_{2}}\left|P_{k_{2}}^{2} * f\right|^{2}\right)^{\frac{1}{2}}\right\|_{L^{p}\left(\mathbb{H}^{1}\right)} \\
& \lesssim\|f\|_{L^{p}\left(\mathbb{H}^{1}\right)}
\end{aligned}
$$


So there remains the estimate of $\mu_{j, k_{2}}^{g l o} * G_{k_{2}}^{2}$. Let us set $\mathcal{L}_{j, k_{2}, L}=L_{j+k_{2}-l_{2}}^{2} *$ $G_{k_{2}}^{2} * L_{j-l_{1}}^{1}$ where $L=\left(l_{1}, l_{2}\right)$. Then our proof is done by the estimates

(6.1) $\|\left(\sum_{j, k_{2}} \mid \mu_{j, k_{2}}^{g l o} *\left[\left.\mathcal{L}_{j, k_{2}, L} * f\right|^{2}\right)^{\frac{1}{2}}\left\|_{L^{p}\left(\mathbb{H}^{1}\right)} \lesssim\right\| f \|_{L^{p}\left(\mathbb{H}^{1}\right)}\right.$,

$$
\|\left(\sum_{j, k_{2}} \mid \mu_{j, k_{2}}^{g l o} *\left[\left.\mathcal{L}_{j, k_{2}, L} * f\right|^{2}\right)^{\frac{1}{2}}\left\|_{L^{2}\left(\mathbb{H}^{1}\right)} \lesssim 2^{-c\left(\left|l_{1}\right|+\left|l_{2}\right|\right)}\right\| f \|_{L^{2}\left(\mathbb{H}^{1}\right)}\right.
$$

(6.1) follows in the same way as (5.2). For (6.2), we use the similar estimate of (5.9) with $\nu=2$ to obtain that

$$
\left\|\widehat{\mu_{j, k_{2}}^{g l o}}(\lambda) \widehat{\mathcal{L}_{j, k_{2}, L}}(\lambda)\right\|_{o p} \lesssim 2^{-c|L|} .
$$

Note that $l_{2} \geq 0$ from the computation of integral kernel of the operator $\widehat{\mu_{j, k_{2}}^{g l o}}(\lambda) \widehat{\mathcal{L}_{j, k_{2}, L}}(\lambda)$. Thus (6.3) combined with (5.4) proves $(6.2)$.

Proof of (3.4). The maximal operator $\sup _{j, k_{2}, k_{3}} \mu_{j, K}^{g l o} * E_{k_{3}-k_{2}}^{1} * f$ is majorized by

$$
\left(\sum_{k^{\prime}}\left|\sup _{j, k_{2}} \mu_{j, k_{2}, k_{2}+k^{\prime}}^{g l o} * E_{k^{\prime}}^{1} * f\right|^{2}\right)^{1 / 2}
$$

where we put $k^{\prime}=k_{3}-k_{2}$. So by using Lemma 1,2 , it suffices to show that

$$
\left\|\sup _{j, k_{2}} \mu_{j, k_{2}, k_{2}+k^{\prime}} * f\right\|_{L^{p}\left(\mathbb{H}^{1}\right)} \lesssim\|f\|_{L^{p}\left(\mathbb{H}^{1}\right)} \cdot
$$

We can easily observe that $\mu_{j, k_{2}, k_{2}+k^{\prime}} * f\left(x_{1}, x_{2}, x_{3}\right)=\mu_{j, k_{2}} * f\left(D_{2,3}^{k^{\prime}}\right)\left(x_{1}, x_{2}, x_{3}-\right.$ $\left.2^{k^{\prime}} x_{2}\right)$ where $D_{2,3}^{k^{\prime}}$ is a diffeomorphism such that $\left.D_{2,3}^{k^{\prime}}\left(x_{1}, x_{2}, x_{3}\right)\right)=\left(x_{1}, x_{2}, x_{3}+\right.$ $2^{k^{\prime}} x_{2}$ ). This observation shows (6.4) with a bound independent of $k^{\prime}$. Hence (3.4) is proved.

\section{References}

[1] A. Carbery, Differentiation in lacunary directions and an extension of the Marinkiewicz multiplier theorem, Ann. Inst. Fourier 38 (1988), 157-168.

[2] H. Carlsson, M. Christ, A. Córdoba, J. Douandikoetxea, J. L. Rubio de Francia, J. Vance, S. Wainger, and D. Weinberg, $L^{p}$ estimates for maximal functions and Hilbert transforms along flat curves in $\mathbb{R}^{2}$, Bull. Amer. Math. Soc. 14 (1986), 263-267.

[3] M. Christ, The strong maximal function on a nilpotent group, Trans. Amer. Math. Soc. 331 (1992).

[4] A. Cordoba, R. Fefferman, On differentiation of integrals, Proc. Nat. Acad. Sci. U.S.A. 74 (1977), 2211-2213.

[5] G. B. Folland, Harmonic Analysis in Phase Space, Annals of Mathematics Studies 122, Princeton Univ.Press (1989).

[6] K. Hare, F.Ricci, Maximal function with polynomial densities in lacunary directions, Tran. Amer. Math. Soc. 355 (2003), 1135-1144.

[7] J. Kim, $L^{p}$-estimate for singular integrals and maximal operators associated with flat curves on the Heisenberg group, Duke. Math. J. 114 (2002), 555-593.

[8] D. Müller, F. Ricci, E. M. Stein, Marcinkiewicz multipliers and multi-parameter structure on the Heisenberg (-type) groups,I, Invent. math 119 (1995), 199-233. 
[9] A. Nagel, E. M. Stein, S. Wainger, Differentiation in lacunary directions, Proc. Nat. Acad. Sci. U.S.A. 75 (1978), 1060-1062.

[10] E. M. Stein, Harmonic Analysis:Real-Variable Methods, Orthogonality, and Oscillatory Integrals, Princeton (1993).

[11] J. -O. Stromberg, Weak estimates for maximal functions with rectangles in certain directions, Ark. f. Mat. 15 (1976), 229-240.

Chung-Ang University Math Department, Huksuk-dong Donjak-Ku, Seoul 156-756, REPUBLIC OF KoREA

E-mail address: jikim@cau.ac.kr 